J. Lake Sci. (湖泊科学), $2007, \mathbf{1 9}(4): 367-372$

http://www. jlakes. org. E-mail: jlakes@ niglas. ac.cn

(c) 2007 by Journal of Lake Sciences

\title{
组合型浮床生态系统的构建及其改善湖泊水源地水质的效果”
}

\author{
李先宁，宋海亮，朱光灿，李大成，吕锡武 \\ (东南大学环境科学与工程系,南京 210096)
}

摘 要: 研究开发了一种由水生植物、水生动物及微生物膜构建的组合型浮床生态系统. 在野外条件下, 考察了该浮床对 富营养化湖泊水在静态条件下的净化效果. 结果表明, $20 \mathrm{~d}$ 内 TN、TP 的去除率分别为 $83.7 \%$ 和 $90.7 \%$,透明度也有大幅 提高. 而且, 组合型浮床系统对有机物尤其是难降解有机物的去除能力较强, 在 $25 \mathrm{~d}$ 内, 对 r-BHC、HE、DDE、DDD、DDT 的 去除率分别为 $25.2 \% 、 63.8 \% 、 42.1 \% 、 71.6 \%$ 和 $27.6 \%$,氯苯、阿特拉津的去除率达 $55.9 \%$ 和 $72.1 \%$. 水生动物的代谢 活动提高了有机物的生物可降解性和氮磷的植物可利用性. 该新型浮床可用于水源地水质改善、污水净化生态工程、富 营养水体的生态恢复等.

关键词 : 浮床; 水生植物; 水生动物;微生物膜; 难降解有机物

\section{Effect of combined floating bed ecosystem on water quality improvement in lake water source area}

LI Xianning, SONG Hailiang, ZHU Guangcan, LI Dacheng \& LU் Xiwu

(Department of Environmental Science and Engineering, Southeast University, Nanjing 210096, P. R. China)

\begin{abstract}
A new type of ecological floating bed is developed that combined hydrophyte, aquatic animal and biofilm together. The purification effect of the floating bed for eutrophic lake water under static condition was tested. Result shows that the removal efficiency of TN and TP is $83.7 \%$ and $90.7 \%$ respectively during 20 days, and the transparency is also heightened sharply. Additionally, the floating bed shows better removal effect on non-degradative organic matters. During 25 days, the removal efficiency of r-BHC, HE, DDE, DDD, DDT can reach 25.2\%, $63.8 \%, 42.1 \%, 71.6 \%$ and $27.6 \%$ separately. Furthermore, for benzene chloride and atraizine, up to $55.9 \%$ and $72.1 \%$ respectively. The biodegradability of non-degradative organic matters and availability of N \& P for hydrophyte are all enhanced by aquatic animal's metabolism. The new type of ecological floating bed may be used to drinking water source quality improvement, ecological engineering for wastewater purification, and ecological restoration of eutrophic lakes.
\end{abstract}

Keywords: Floating bed; hydrophyte; aquatic animal; biofilm; non-degradative organic matter

生物浮床技术将水生植物或部分陆生植物固定在浮床上,通过植物对氮、磷等营养物的吸收作用, 根系 附着微生物对污染物的降解作用, 达到去除氮、磷等营养物, 有效控制水体富营养化的目的. 生物浮床具有 直接从水体中去除污染物, 充分利用水面而无需占用土地等特点; 能够在各种水深条件的水体中应用, 且造 价低廉, 运行管理相对容易 ${ }^{[1,2]}$. 目前,生物浮床技术在我国的研究与应用日益增多, 规模也已由试验室研 究开始走向规模性示范和应用 ${ }^{[3]}$. 但大部分浮床的净化主体仍仅仅是植物, 研究重点也主要是对比不同植 物去除氮、磷等营养物的能力 ${ }^{[4,5]}$, 但生物量的限制使得仅通过篮选植物种类已不可能使净化效果有根本 性的提高, 因此必须在浮床的构造形式上有所突破. “生物共生机制、生物多样性及食物链原理” 是生态工 程重要的基本原理,通过人工构筑共生生态机制和食物链的 “加环” 可以大幅度提高生态效应和生态净化

* 国家 “十五”重大科技专项项目 (2002AA601011) 和 教育部留学回国人员科研启动基金共同资助. $2006-07-04$ 收稿; 2006-10-24 收修改稿. 李先宁, 男,1964 年生, 博士, 副教授; E-mail:lxn@ seu. edu. cn. 
功能. 本研究在普通生物浮床的基础上, 通过对水生植物、水生动物及微生物生态系统的合理构建, 开发出 一种组合型新生态浮床,并研究了其改善富营养化湖泊水源地水质的功能.

\section{1 试验条件、材料、方法}

\section{1 组合型生态浮床的设计}

组合型生态浮床如图 1 所示, 整体为 $1 \mathrm{~m}($ 长) $\times 1 \mathrm{~m}$ (宽) $\times 1.1 \mathrm{~m}$ (高)的长方体构造, 设计成上、中、下 三层结构. 上层区域 ( I ) 为水生植物区,种植水生经济植物,并通过合理设置根区空间使植物根系形成“毡

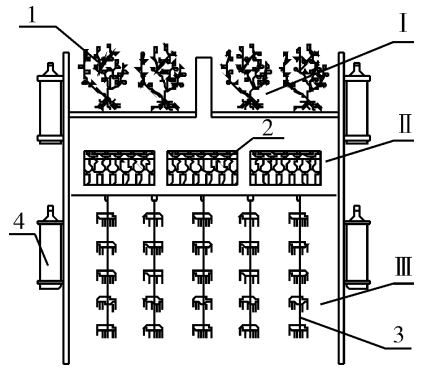

图 1 组合型生态浮床示意图 $\mathrm{I}$ : 水生植物区, II : 水生动物区,

III : 人工介质区 $(1$ : 空心菜,

2 : 笼养河蚬, 3 : 人工介质, 4 : 浮桶)

Fig. 1 Schematic diagram of ecological combination floating bed 垫” 状构造, 既不影响植物的吸收功能, 又提高了根系截留颗粒性 污染物和藻类的能力, 该区域有效高为 $20 \mathrm{~cm}$. 中层区域 (II) 为水 生动物区, 笼养滤食性水生动物贝类, 利用贝类的滤食作用去除污 染物, 并通过贝类的消化作用大幅度提高有机污染物的生物可降 解性. 笼网采用渔网制作成双层结构, 既能满足对贝类生物量需 要, 又避免了贝类过度堆积, 保证其成活率, 该区域高为 $30 \mathrm{~cm}$. 下 层区域 (III) 为人工介质区, 悬挂兼具软性及半软性特征的高效人 工介质, 大量富集微生物, 形成高效生物膜净化区, 该部分高为 $60 \mathrm{~cm}$.

\section{2 试验条件与方法}

为了研究组合型生态浮床对富营养化水体水质的净化效果, 在无锡市太湖梅梁湾之滨, 建设 $3.6 \mathrm{~m}$ (长) $\times 2 \mathrm{~m}$ (宽) $\times 1.8 \mathrm{~m}$ (高) 的水泥砖砌封底试验水池 2 个,在其中 1 个水池中部放置上 述组合型生态浮床, 浮床面积覆盖率 $13.6 \%$; 另 1 个水池则为空白 对照池, 湖水经提升水洜平行进人各个试验水池.

2005 年 5 月中旬开始水生动物驯养、植物移栽和人工介质自然挂膜, 同年 7 月开始相关试验研究. 试 验期间上层水生植物区种植空心菜 (Ipomea aquatica), 中层水生动物区养殖河蚬 (Corbicula fluminea). 空心 菜取自田间,清洗根系上附着的土壤, 称重后按相同重量 $(3000 \mathrm{~g})$ 移植于浮床,试验期间空心菜在浮床上已 经生长良好. 河蚬购于无锡贡湖湾茶场, 清洗后放置于浮床内的河蚬笼内, 每层笼高度 $10 \mathrm{~cm}$, 共放养河蚬 350 只,每层放养密度为 175 只 $/ \mathrm{m}^{2}$. 河蚬笼与植物层之间的净空高度为 $10 \mathrm{~cm}$. 浮床内共挂组合介质 81 串, 每串上有盘片 8 片, 填料盘片间隔为 $7 \mathrm{~cm}$. 试验期间水池内水温较为稳定, 在 $30-33^{\circ} \mathrm{C}$, 水池中 $\mathrm{pH}$ 值在 7.6-8.4 之间, 与湖区基本一致. 待生态浮床稳定运行后,将 2 个试验水池同时重新换水,浮床日处理水量 为 $1.1 \mathrm{~m}^{3}$, 则空床水力停留时间为 $1 \mathrm{~d}$, 进行静态试验.

\section{3 水质测定方法}

每间隔 $5 \mathrm{~d}$ 取水样测定水质,取样时间均为上午 10 时左右. 从水池四角和中央共 5 点各取 $500 \mathrm{ml}$ 水样 后混合,供水质分析使用.

$\mathrm{TN} 、 \mathrm{NH}_{4}^{+}-\mathrm{N} 、 \mathrm{NO}_{3}^{-}-\mathrm{N} 、 \mathrm{NO}_{2}^{-}-\mathrm{N}$ 和高锰酸盐指数 $\left(\mathrm{COD}_{\mathrm{Mn}}\right)$ 均按国家标准方法 ${ }^{[6]}$ 测定, TOC 采用 $\mathrm{TOC}-\mathrm{V}_{\mathrm{CSH}}$ (日本岛津) 测定, DO 采用碘量法, $\mathrm{pH}$ 值采用 TOA-HM-14P 仪 (日本东亚) 测定, Chl. $a$ 采用丙酮提取 - 分光 光度计测定法.

对水样中的有机氯类、氯苯和阿特拉津进行气相色谱分析. 取 $1 \mathrm{~L}$ 混合水样经 $\mathrm{GF} / \mathrm{C}$ 滤膜过滤后, 以 $0.5 \mathrm{~L} / \mathrm{h}$ 的速度通过预先活化的 C18 固相萃取柱 (Supelco 公司,美国)进行富集,用选择性溶剂进行洗脱、定 容后, 有机氯类、氯苯采用 GC-ECD 测定, 阿特拉津采用 GC-NPD 测定, 气相色谱仪为 Agilent 6890 (HewlettPackard 公司, 美国),色谱条件参照相关标准方法 ${ }^{[6]}$.

\section{2 结果与分析}

\section{1 氮的去除}

图 2 分别表示了试验水池中 $\mathrm{TN} 、 \mathrm{NH}_{4}^{+}-\mathrm{N} 、 \mathrm{NO}_{3}^{-}-\mathrm{N}$ 浓度随时间的变化. 
空白池与浮床池的进水 TN 浓度分别为 $7.35 \mathrm{mg} / \mathrm{L}$ 和 $8.06 \mathrm{mg} / \mathrm{L}$. 在试验期的前 $20 \mathrm{~d}$ 浮床池中的 TN 浓 度基本呈直线下降至 $1.31 \mathrm{mg} / \mathrm{L}$, 去除率为 $83.7 \%$, 单位浮床面积的 $\mathrm{TN}$ 去除速率为 $3.98 \mathrm{~g} /\left(\mathrm{m}^{2} \cdot \mathrm{d}\right)$. 当 $\mathrm{TN}$ 浓度低于 $1 \mathrm{mg} / \mathrm{L}$ 后, 浓度变化梯度降低, 表明当水中 $\mathrm{TN}$ 浓度小于 $1 \mathrm{mg} / \mathrm{L}$ 时, 生态浮床的 $\mathrm{TN}$ 去除速率 减小. 在 $25 \mathrm{~d}$ 内的 TN 去除率为 $86.2 \%$. 空白池 TN 浓度也呈局部下降趋势, 但下降幅度明显低于浮床池. $20 \mathrm{~d}$ 内的 TN 去除率为 $42.5 \%$. 空白池的 TN 去除主要缘于含氮颗粒物的自然沉降以及水体悬浮微生物的 硝化与反硝化作用 (图 2a).
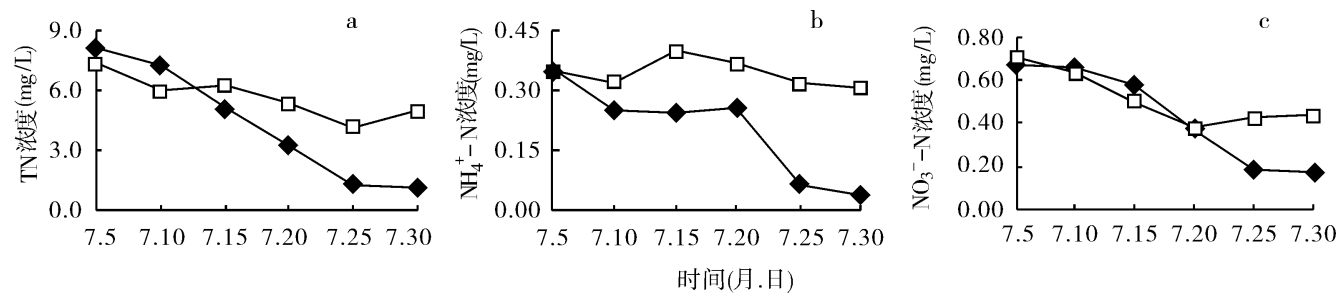

图 2 不同池中各存在形态氮随时间的变化

Fig. 2 Changes of different nitrogen species concentration in different tanks with time

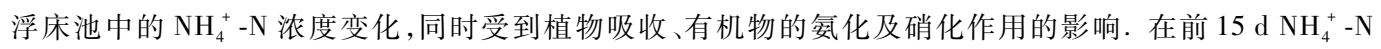
浓度下降缓慢, 这是由于有机物氨化作用较强, 有机氮转化为氨氮, 造成表观 $\mathrm{NH}_{4}^{+}-\mathrm{N}$ 浓度下降较缓慢; 随着 有机氮的降解及硝化作用的增强, 后期 $\mathrm{NH}_{4}^{+}-\mathrm{N}$ 急剧下降, $25 \mathrm{~d}$ 内的去除率达到 $88.6 \%$, 而空白池 $\mathrm{NH}_{4}^{+}-\mathrm{N}$ 去除率仅为 $10.7 \%$ (图 2b).

浮床池与空白池的 $\mathrm{NO}_{3}^{-}-\mathrm{N}$ 总去除率分别为 $74.3 \%$ 和 $39 \%$. 在试验开始后的前 $15 \mathrm{~d}$ 两系统中的 $\mathrm{NO}_{3}^{-}-\mathrm{N}$ 表观浓 度均呈下降趋势, 且下降速率接近, $15 \mathrm{~d}$ 后浮床池继续下 降至 $0.17 \mathrm{mg} / \mathrm{L}$, 而空白池则呈微小上升趋势. 在浮床池中 $\mathrm{NO}_{3}^{-}-\mathrm{N}$ 在生态浮床的各种净化作用 (植物吸收, 植物根系 及人工介质附着微生物的硝化、反硝化作用等)下得到有 效去除, 而空白池中 $\mathrm{NO}_{3}^{-}-\mathrm{N}$ 的浓度下降, 主要依靠池中藻 类和其他微生物增殖的同化吸收. $\mathrm{NO}_{2}^{-}-\mathrm{N}$ 浓度的变化情 况基本与 $\mathrm{NO}_{3}^{-}-\mathrm{N}$ 基本一致,浮床池的去除率为 $82.3 \%$, 空 白池为 $8.1 \%$ (图 2c).

\section{2 磷的去除}

图 3 表示了试验水池中 TP 浓度随时间的变化, 在试

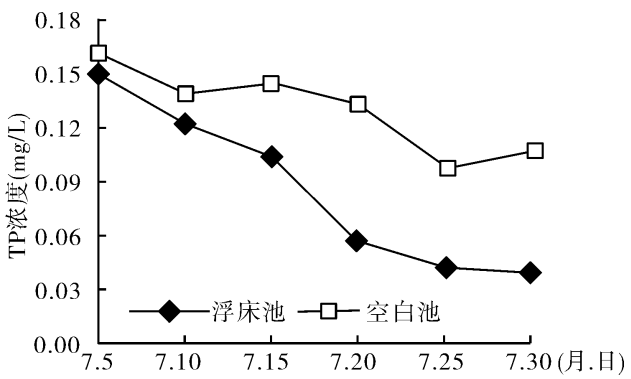

图 3 不同池中总磷的变化

Fig. 3 Change of TP concentration in different tanks 验开始的前 $20 \mathrm{~d}$, 浮床池的去除率为 $90.7 \%$, 空白池为 $40.0 \%$. 可见浮床池的 TP 去除率达到了较高水平. 同时如图所示, 浮床池在 TP 为 $0.06 \mathrm{mg} / \mathrm{L}$ 以上这样的低浓度条件下其浓度下降仍然显著,表明组合型生态 浮床对较低浓度的富营养水体可以取得良好的净化效果, 单位浮床面积的 TP 去除速率为 $0.08 \mathrm{~g} /\left(\mathrm{m}^{2}\right.$. d).

有关生物浮床去除氮、磷的相关研究报道中, 宋祥甫等 ${ }^{[7]}$ 采用水稻生物浮床,在覆盖率为 $20 \%, 84 \mathrm{~d}$ 的 试验条件下, 取得了 TKN 净去除率为 $29.0 \%$, TP 净去除率为 $32.1 \%$ 的结果 (若不扣除空白对照, 计算得 TKN 、TP 去除率分别为 $31.4 \% 、 32.5 \%)$; 经计算, 单位浮床面积的 TKN 和 TP 去除速率分别为 $0.05 \mathrm{~g} /\left(\mathrm{m}^{2}\right.$ $\cdot \mathrm{d})$ 和 $0.007 \mathrm{~g} /\left(\mathrm{m}^{2} \cdot \mathrm{d}\right)$. 邴旭文等 ${ }^{[8]}$ 应用美人蕉生物浮床净化富营养化池塘水, 在覆盖率为 $20 \%$ 的条件 下, $25 \mathrm{~d}$ 时 TN 去除率约为 $60 \%$, TP 去除率约为 $30 \%$; 经计算, 单位浮床面积的 TN 和 TP 去除速率分别为 $1.6 \times 10^{-4} \mathrm{~g} /\left(\mathrm{m}^{2} \cdot \mathrm{d}\right)$ 和 $1.1 \times 10^{-5} \mathrm{~g} /\left(\mathrm{m}^{2} \cdot \mathrm{d}\right)$. 马立珊等 ${ }^{[9]}$ 应用香根草生物浮床对城市河道水进行净化 试验研究的结果表明, $25 \mathrm{~d}$ 时 TN 去除率约小于 $20 \%$, TP 去除率小于 $30 \%$; 计算得到 TN、TP 单位去除速率 分别为 $0.003 \mathrm{~g} /\left(\mathrm{m}^{2} \cdot \mathrm{d}\right)$ 和 $0.0009 \mathrm{~g} /\left(\mathrm{m}^{2} \cdot \mathrm{d}\right)$. TN、TP 的去除率和去除速率均小于本试验, 因此组合型生 
态浮床对 $N 、 P$ 的去除效果比以植物为主体的普通生物浮床有大幅提高.

\section{3 藻类的去除}

Chl. $a$ 是估算浮游植物生物量 (本文主要指藻类) 的重要指标. 图 4 表示了试验水池中 Chl. $a$ 浓度随时 间的变化. 试验开始时浮床池及空白池的 Chl. $a$ 初始浓度为 $24.23 \mu \mathrm{g} / \mathrm{L}$, 试验开始 $5 \mathrm{~d}$ 内, 浮床池的 Chl. $a$ 浓度急剧下降至 $5.71 \mu \mathrm{g} / \mathrm{L}$, 去除率为 $76.4 . \%$, 之后 Chl. $a$ 浓度变化趋缓,在试验结束时第 $25 \mathrm{~d}$ 去除率可达 $89.5 \%$. 而空白池出现了 Chl. $a$ 浓度的上升现象, 在试验开始后的第 $20 \mathrm{~d}$ 上升至 $47.74 \mu \mathrm{g} / \mathrm{L}$, 与初始值相 比上升了约 2 倍. 与空白池相比,一方面浮床的截留吸附作用去除了一部分藻类, 另一方面浮床的综合作 用使水体中的氮磷营养盐浓度大大降低, 浮床植物在与藻类的竞争中也处于优势地位, 此外植物化感作用 对藻类也有一定的抑制作用 ${ }^{[10,11]}$, 导致藻类无法繁殖. 因此几方面的原因使浮床池中的 Chl. $a$ 浓度迅速降 低并始终维持在较低水平. 而空白池中, 氮磷浓度缓慢下降, 且维持在相对较高水平, 在没有其他植物竞争 的情况下藻类自然增殖, 于是出现了 Chl. $a$ 浓度升高的现象. 可见, 组合型生态浮床对于降低水体藻类含 量具有良好的效果.

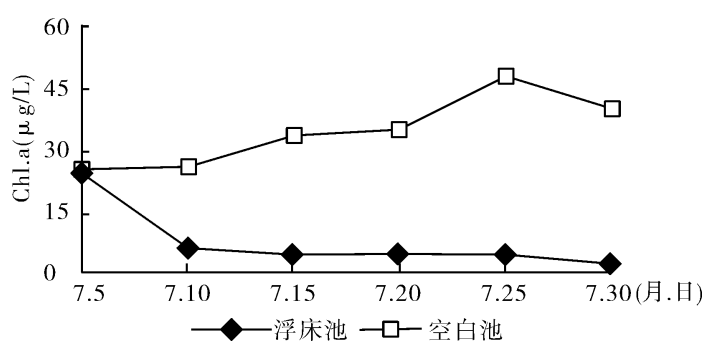

图 4 不同池中 Chl. $a$ 浓度的变化

Fig. 4 Change of Chl. $a$ concentration in different tanks

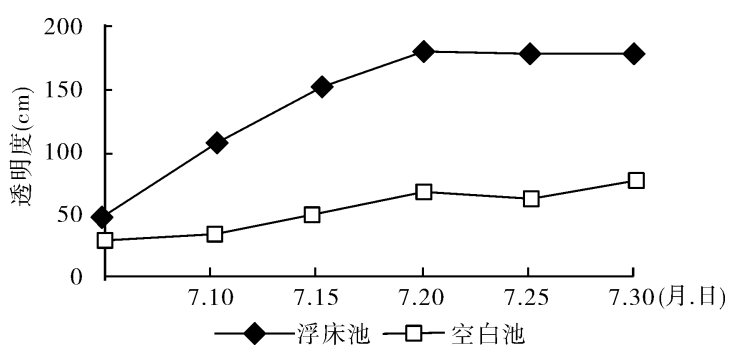

图 5 不同池中透明度的变化

Fig. 5 Change of transparence in different tanks

透明度是表示各种水体能见程度的一个量度, 也是水体混浊程度的标志. 光是水体初级生产力的能 源, 水体透明度是光通量沿水深分布的量度, 透明度下降可进一步引起水生生态系统破坏 ${ }^{[12]}$. 图 5 表示了 透明度随时间的变化, 浮床池由于生态浮床的净化作用, 在 $15 \mathrm{~d}$ 内将透明度由 $46 \mathrm{~cm}$ 提高到超过 $180 \mathrm{~cm}$, 已 经清澈见底. 透明度的提高说明悬浮物、浮游植物得到了有效的去除, 这一点与 Chl. $a$ 的去除效果相吻合. 透明度的提高可改善水中浮游动物的生境, 提高浮游动物生物量, 丰富生物多样性, 为沉水植物乃至水体自 净能力的恢复提供了前提 ${ }^{[13]}$.

\section{4 有机物的去除}

图 6 表示了 COD 浓度随时间的变化,试验开始后的 $15 \mathrm{~d}$ 内浮床池的 COD 浓度基本呈直线型下降,之 后趋于平缓,第 $15 \mathrm{~d}$ 时浓度减少到 $4.2 \mathrm{mg} / \mathrm{L}$, 去除率为 $44.4 \%$. 而空白池在前 $5 \mathrm{dCOD}$ 浓度略有下降, 之后 受藻类增长的影响而呈回升趋势, 在第 $25 \mathrm{~d}$ 时浓度为 $7.16 \mathrm{mg} / \mathrm{L}$, 总去除率仅为 $9.5 \%$.

在类似研究中有关 Chl. $a$ 和 COD 去除效果的报道较少, 邴旭文等 ${ }^{[8]}$ 采用种植美人蕉的生物浮床, 在与 本研究相近的覆盖率条件下 (覆盖率 20\%), 试验开始 $7 \mathrm{~d}$ 内 Chl. $a$ 的去除率小于 $10 \%, 63 \mathrm{~d}$ 后为 $54 \%$ 左右, COD 去除率 $15 \mathrm{~d}$ 时小于 $8 \%$. 李欲如等 ${ }^{[14]}$ 在水䔨菜生物浮床小试研究中 COD 去除率为 $37 \%$. 上述两项研 究中的 COD 起始浓度在 8-15 mg/L, 在起始浓度高于本试验条件的情况下, 去除率均低于本试验结果, 可 见组合型生态浮床在去除率上明显高于普通生物浮床.

表 1 对比了难降解性微量有机物在浮床池和空白池中的浓度变化. 在浮床池中,有机氯农药类物质浓 度均有显著下降, r-BHC、HE、DDE、DDD、DDT 的去除率分别为 $25.2 \% 、 63.8 \% 、 42.1 \% 、 71.6 \%$ 和 $27.6 \%$; 而 空白池中除DDD、DDE 浓度有一定降低外, 其余三种有机氯农药类物质浓度不降反升. 浮床池中氯苯、阿 特拉津的去除率达 $55.9 \%$ 和 $72.1 \%$, 而空白池则分别为 $1.8 \%$ 和 $11.2 \%$. 由上可见,浮床池对多种难降解 有机物的去除效果大大优于空白池. 
表 1 浮床池和空白池中难降解微量有机物浓度的变化

Tab. 1 The change of refractory micro organic matters in tank with and with out floating bed

\begin{tabular}{|c|c|c|c|c|c|c|c|}
\hline & \multicolumn{5}{|c|}{ 有机氯农药类 (ng/L) } & \multirow{2}{*}{$\begin{array}{c}\text { 氯苯 } \\
(\mu \mathrm{g} / \mathrm{L})\end{array}$} & \multirow{2}{*}{$\begin{array}{c}\text { 阿特拉津 } \\
(\mathrm{ng} / \mathrm{L})\end{array}$} \\
\hline & $\mathrm{r}-\mathrm{BHC}$ & $\mathrm{HE}$ & DDE & DDD & DDT & & \\
\hline 初始浓度 (7 月 5 日) & 0.457 & 0.047 & 0.019 & 1.560 & 0.141 & 0.218 & 96.79 \\
\hline 终了浓度 & 0.342 & 0.017 & 0.011 & 0.443 & 0.102 & 0.096 & 27.04 \\
\hline (7月 30 日) & 1.800 & 0.172 & 0.011 & 1.090 & 0.159 & 0.214 & 85.92 \\
\hline
\end{tabular}

综上所述, 通过生态系统的合理构建以及食物链的作用, 可以大幅度提高浮床去除藻类和有机物的效 果, 尤其是对难降解有机物的去除能力显著增强,这对于改善水源地水质具有特别重要的意义.

\section{3 讨论}

目前富营养化湖泊、水源地以及缓流河道的水质改 善技术大致分为工程性措施、化学方法以及生物强化 法, 其中生物强化法主要采用水生植物技术、水生动物 技术以及微生物技术, 本文中的组合型生态浮床, 通过 将三种生物强化净化技术在有限的空间内有机结合, 人 工构建小型生态系, 使浮床在提高水体透明度、去除氮 磷营养盐、降解有机物等方面的效果显著增强. 生态浮 床对有机物及氮、磷等营养物净化能力的显著提高, 主 要得益于生态浮床中生态系统的合理构筑, 通过水生植 物, 水生动物及微生物所构成的食物链强化得以实现. 试验结束时空心菜鲜重由 $3 \mathrm{~kg}$ 增加至 $4.5 \mathrm{~kg}$, 河蚬在初

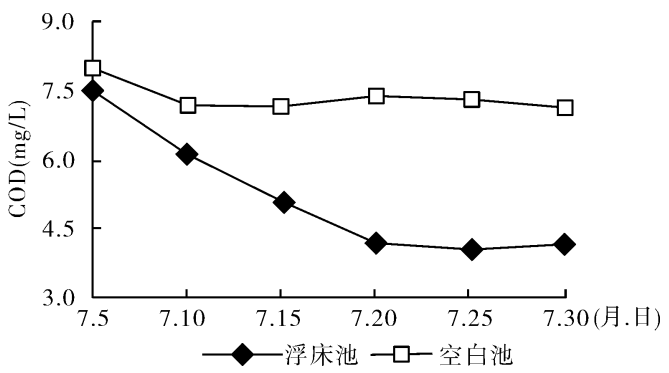

图 6 不同池中 COD 的变化

Fig. 6 Change of COD concentration in different tanks 始密度为 175 只 $/ \mathrm{m}^{2}$ 条件下成活率约为 $80 \%$. 其中应用 生态工程的 “加环” 原理,在生态净化系统中引人水生动物一一河蚬的环节,大幅度提高了有机物的可生化 性是提高净化效率的关键之一. 试验结果表明, 河蚬对太湖原水可生化性指标 $\mathrm{B} / \mathrm{C}$ 比的提高效果明显, 放 养河蚬 $1 \mathrm{~d}$ 后, 可将可生化性较差的湖水 $\mathrm{B} / \mathrm{C}$ 比从 0.18 提高到 0.87 , 同时水中的氨氮浓度也明显提高 (另 文报道). 河蚬为滤食型水生动物, 通过吸管伸在水中进行呼吸和摄取饵料, 将悬浮于水中的有机颗粒物, 藻类等摄人体内, 部分消化吸收, 部分以粪便的形式排放,除通过同化作用净化水质外, 消化作用促进了不 溶性有机颗粒的可溶性和可生化性以及氨化作用. 难降解性有机物生物可降解性及氨化作用的提高为生 态浮床下部人工介质附着微生物膜提供了优质的基质条件, 从而促进了微生物的生物活性, 使微生物原来 无法有效降解的有机物得到去除, 提高了浮床整体对难降解有机物和藻类的去除效果. 氨化作用的提高还 进一步促进了生物膜中硝化反应进程, 提高了生态浮床的生物脱氮功能, 同时也为水生植物吸收氮创造了 有利条件.

\section{4 结论}

(1) 在组合型生态浮床面积覆盖率为 $13.6 \%$ 的水池中, 原水停留 $20 \mathrm{~d}$ 后, TN 、 TP 的去除率分别为 $83.7 \%$ 和 $90.7 \%$, 单位面积浮床对 $\mathrm{N}, \mathrm{P}$ 的去除速率分别为 $3.98 \mathrm{~g} /\left(\mathrm{m}^{2} \cdot \mathrm{d}\right)$ 和 $0.08 \mathrm{~g} /\left(\mathrm{m}^{2} \cdot \mathrm{d}\right)$.

（2）在有生态浮床覆盖的水池中, $25 \mathrm{~d}$ 内 Chl. $a$ 去除率可达 $89.5 \%$, 水体透明度由 $46 \mathrm{~cm}$ 提高到超过 $180 \mathrm{~cm}$.

(3) 组合型生态浮床对原水中的有机物也有较好的去除效果; 尤其对于难降解有机物有较强的去除能 力, 在 $25 \mathrm{~d}$ 内对 $\mathrm{r}-\mathrm{BHC} 、 \mathrm{HE} 、 \mathrm{DDE} 、 \mathrm{DDD} 、 \mathrm{DDT}$ 的去除率分别为 $25.2 \% 、 63.8 \% 、 42.1 \% 、 71.6 \%$ 和 $27.6 \%$, 氯 苯、阿特拉津的去除率达 $55.9 \%$ 和 $72.1 \%$. 水生动物的消化作用提高了有机物的生物可降解性. 


\section{5 参考文献}

［1］中村圭吾,島谷幸広. 人工浮島の機能と技術の現狀. 土木技術資料, 1999,41(7):26-31.

[2] Nakamura K, Shimatani Y. Water purification and environmental enhancement by the floating wetland. Proceeding of $6^{\text {th }}$ IAWQ Asia-Pacific regional conference in Korea, 1997.

[3] 周小平, 王建国,薛利红等. 浮床植物系统对富营养化水体中氮、磷净化特征的初步研究. 应用生态学 报,2005,16(11):2199-2203.

[4] 黎华寿, 聂呈荣, 方文杰等. 浮床栽培植物生长特性的研究. 华南农业大学学报(自然科学版), 2003, 24(2): $12-15$.

[5]卢进登,帅方敏,赵丽娅等. 人工生物浮床技术治理富营养化水体的植物遴选. 湖北大学学报(自然科 学版),2005,27(4):402-404.

[6]国家环保总局. 水和废水监测分析方法 (第 4 版). 北京: 中国环境科学出版社,2003.

[7] 宋祥甫, 邹国燕, 吴伟明等. 浮床水稻对富营养化水体中氮、磷的去除效果及规律研究. 环境科学学 报, $1998, \mathbf{1 8}(5): 489-494$.

[8] 邴旭文,陈家长. 浮床无土栽培植物控制池塘富营养化水质. 湛江海洋大学学报, 2001,21 (3):29 -33 .

[9] 马立珊, 骆永明, 吴龙华等. 浮床香根草对富营养化水体氮磷去除动态及效率的初步研究. 土壤, 2000, $32(2): 99-101$.

[10] Nakai S, Inoue Y, Hosomi M et al. Growth inhibition of blue-green algae by allelopathic effects of marophytes. Water Science and Technology,1999,39(8):47 - 53.

[11] Li F M, Hu H Y. Allelopathic effects of different macrophytes on the growth of Microcystis aeruginosa. Allelopathy Journal,2005,15(1):145 - 152 .

[12] Gulati S D, Van D E. Lakes in the Netherlands, their origin, eutrophication and restoration: state of the art review. Hydrobologia, $2002, \mathbf{4 7 8}(1): 73-106$.

[13] Havens K E, Sharfstein B, Brady M A et al. Recovery of submerged plants from high water stress in a large subtropical lake in Florida, USA. Aquatic Botany,2004,78(1):67 - 82.

[14] 李欲如, 操家顺,徐峰等. 水䔨菜对苏州重污染水体净化功能的研究. 环境污染与防治, 2006, 28 (1) $: 69-71$. 